\author{
ANNALES \\ POLONICI MATHEMATICI \\ $99.2(2010)$
}

\title{
Timelike Christoffel pairs in the split-quaternions
}

\author{
by M. P. Dussan (São Paulo) and M. Magid (Wellesley, MA)
}

\begin{abstract}
We characterize the Christoffel pairs of timelike isothermic surfaces in the four-dimensional split-quaternions. When restricting the receiving space to the threedimensional imaginary split-quaternions, we establish an equivalent condition for a timelike surface in $\mathbb{R}_{2}^{3}$ to be real or complex isothermic in terms of the existence of integrating factors.
\end{abstract}

1. Introduction. Timelike isothermic surfaces have been studied by the authors and others $([2,[3],[11,[12,3]$, 7]). The chief difference in this setting is that there are two types of isothermic surfaces, real isothermic and complex isothermic ones, depending on whether the shape operators are diagonalizable over $\mathbb{R}$ or $\mathbb{C}$. Just as in the Riemannian case, the geometric transformations of timelike isothermic surfaces are known to be important tools in the understanding of their geometry and their relationship with integrable systems.

The purpose of this paper is to study the Christoffel pairs of real and complex isothermic surfaces in codimension two and one, describing those in the split-quaternionic setting. Our study was motivated by Hertrich-Jeromin's work in [6], where an excellent study of the Christoffel pairs of isothermic surfaces in codimension two was made utilizing the quaternions $\mathbb{H}$, and the Riemann surface structure of the surface. His definition of Christoffel transform is a generalization of the definition of Christoffel transforms for isothermic surfaces in $\mathbb{R}^{3}$. However when the ambient space is $\mathbb{H}=\mathbb{R}^{4}$, he observes that the Christoffel pair constructed using his quaternionic characterization generally does not share the same tangent planes. With an adjustment of the definition, this defect can be removed.

In this paper we start by looking at the codimension-two case for timelike isothermic surfaces in $\mathbb{H}^{\prime}=\mathbb{R}_{2}^{4}$, the split-quaternions. We characterize the real and complex isothermic surfaces in terms of the closure of suit-

2010 Mathematics Subject Classification: Primary 53A30; Secondary 53C50. Key words and phrases: timelike surface, Christoffel pairs, split-quaternions. 
able $\mathbb{H}^{\prime}$-valued 1-forms and we prove that the corresponding immersions determined by these forms are also timelike isothermic surfaces of real and complex type, respectively. Then when taking the split-quaternionic conjugate of one of those surfaces, we show that the new surface pair constitutes a classical Christoffel pair. In addition, we give symmetric conditions to construct Christoffel pairs in $\mathbb{H}^{\prime}$. For the particular case that the receiving space is the imaginary split-quaternions, we obtain an equivalent condition for a timelike surface in $\mathbb{R}_{2}^{3}$ to be real or complex isothermic, in terms of the existence of integrating factors for an expression involving the mean curvature and the Gauss map. That equivalent condition shows, for instance, that whenever one of the timelike surfaces of a Christoffel pair is a minimal surface, the other is a scaling of its Gauss map. Also the Christoffel transform of a surface of constant mean curvature $H \neq 0$ is a parallel surface of the same constant mean curvature. Finally, we characterize the Hopf differential for real and complex isothermic surfaces via a split-complex variable.

The split-quaternions $\mathbb{H}^{\prime}$ were first discovered in [9] and [10]. These form a real, four-dimensional associative algebra containing some non-invertible, non-zero elements. They were used in [12] and a thorough treatment can be found in [7, which also contains an introduction to the split-complex numbers $\mathbb{C}^{\prime}$ defined as $\left\{s+k^{\prime} t \mid k^{\prime 2}=1, s, t \in \mathbb{R}\right\}$. The split-complex numbers appear naturally when looking at immersions in $\mathbb{H}^{\prime}$, which can be seen, for example, in the proof of Theorem 2.2 in this note.

The organization of this paper is as follows. In Section 2 we establish some basic results involving the algebra of split-quaternions and the description that produces the normal vector in $\mathbb{R}_{2}^{3}$. It also contains the results characterizing the Christoffel pairs of timelike isothermic surfaces in $\mathbb{H}^{\prime}=\mathbb{R}_{2}^{4}$. Section 3 is devoted to the codimension-one case when restricting to the imaginary split-quaternions.

2. Surfaces in $\mathbb{H}^{\prime}$. Our timelike surfaces will sit in $\mathbb{H}^{\prime}$, a four-dimensional Clifford algebra given by

$$
\mathbb{H}^{\prime}=\left\{x_{0}+x_{1} i+x_{2} j^{\prime}+x_{3} k^{\prime} \mid x_{0}, x_{1}, x_{2}, x_{3} \in \mathbb{R}\right\}
$$

with

$$
\begin{gathered}
i^{2}=-1, \quad j^{\prime 2}=1=k^{\prime 2}, \\
i j^{\prime}=k^{\prime}, \quad j^{\prime} i=-k^{\prime}, \quad j^{\prime} k^{\prime}=-i, \quad k^{\prime} j^{\prime}=i, \quad i k^{\prime}=-j^{\prime}, \quad k^{\prime} i=j^{\prime} .
\end{gathered}
$$

This was defined by Libermann in [9], and was called a quaternionic algebra of the second type. We will call them split-quaternions. For $x, y \in \mathbb{H}^{\prime}$ we define our inner product $x \cdot y$ by

$$
x \cdot y=\operatorname{Re}(\bar{x} y)=x_{0} y_{0}+x_{1} y_{1}-x_{2} y_{2}-x_{3} y_{3},
$$


where $\overline{x_{0}+x_{1} i+x_{2} j^{\prime}+x_{3} k^{\prime}}=x_{0}-x_{1} i-x_{2} j^{\prime}-x_{3} k^{\prime}$. The metric chosen here in $\mathbb{H}^{\prime}$ has signature $(2,2)$. We call those split-quaternions with $x_{0}=0$ imaginary, and denote them by $\operatorname{Im} \mathbb{H}^{\prime}$. They inherit a metric of signature $(2,1)$ from $\mathbb{H}^{\prime}$ and are identified in all that follows with $\mathbb{R}_{2}^{3}$. Thus, when we discuss surfaces in three-space, we are working with the negative of the standard metric on Lorentz three-space used in [12, but it is the standard metric on the split-quaternions. Note that if $x \in \operatorname{Im} \mathbb{H}^{\prime}$ then $\bar{x}=-x$, and that $x^{-1}=\frac{\bar{x}}{x \cdot x}$.

For imaginary $x$ and $y$ an important formula for calculations is

$$
x y=-x \cdot y-x \times y .
$$

Here $x \times y$ is the cross product in $\mathbb{R}_{2}^{3}$ given by

$$
(x \times y) \cdot z=\operatorname{Det}[z, x, y] .
$$

We can define, exactly as in [8], a wedge product on $\mathbb{H}^{\prime}$-valued one-forms on $M_{1}^{2}$ by

$$
\alpha \wedge \beta(X, Y)=\alpha(X) \beta(Y)-\alpha(Y) \beta(X),
$$

which satisfies the identities

$$
\begin{aligned}
\overline{\alpha \wedge \beta} & =-\bar{\beta} \wedge \bar{\alpha}, & \alpha \wedge h \beta & =\alpha h \wedge \beta, \\
d(h \alpha) & =d h \wedge \alpha+h d \alpha, & d(\alpha h) & =d \alpha h-\alpha \wedge d h,
\end{aligned}
$$

where $h: M_{1}^{2} \rightarrow \mathbb{H}^{\prime}$. We also identify two-forms on $M_{1}^{2}$ with their quadratic forms by

$$
\omega(X)=\omega\left(X, J^{\prime} X\right)
$$

for $\omega$ an $\mathbb{H}^{\prime}$-valued two-form on $M_{1}^{2}$, and $J^{\prime}$ an integrable product structure satisfying $J^{\prime 2}=\mathrm{Id}$, intrinsic to the Lorentz surface $M_{1}^{2}$, which we define by choosing the two future directed null vectors $\partial_{x}, \partial_{y}$ in the chosen time cone so that $\left\{\partial_{x}, \partial_{y}\right\}$ is positively oriented and with $J^{\prime}(\partial x)=-\partial x, J^{\prime}(\partial y)=\partial y$. If we define $* \alpha=\alpha \circ J^{\prime}$ then we have

$$
\alpha \wedge \beta=\alpha(* \beta)-(* \alpha) \beta,
$$

since $(\alpha \wedge \beta)\left(U, J^{\prime} U\right)=\alpha(U) \beta\left(J^{\prime} U\right)-\alpha\left(J^{\prime} U\right) \beta(U)$. Note that in the Lorentzian setting we have $*^{2}=\mathrm{Id}$. There is an algebraic lemma whose statement and proof is quite similar to one in [1].

Lemma 2.1 (Algebra of split-quaternions). For $x, y \in \mathbb{H}^{\prime}$ :

1. $x y=y x$ iff $\operatorname{Im}(x)$ and $\operatorname{Im}(y)$ are linearly dependent over $\mathbb{R}$.

2. $\operatorname{Im}\left(x^{2}\right)=0$ iff $x$ is either real or purely imaginary.

3. $x^{2}=1$ iff $x= \pm 1$ or $x$ is purely imaginary and $x \cdot x=-1$.

4. $x^{2}=-1$ iff $x$ is purely imaginary and $x \cdot x=1$.

We also need the following version of Lemma 2.1 found in [8]. 
Lemma 2.2. $f: M_{1}^{2} \rightarrow \mathbb{R}_{2}^{3}$ is a conformal immersion iff there exists $N: M_{1}^{2} \rightarrow \mathbb{H}^{\prime}$ such that

$$
(* d f) U=d f\left(J^{\prime} U\right)=N d f(U) .
$$

If $(\dagger)$ holds then $N: M_{1}^{2} \rightarrow H_{1}^{2} \subset \mathbb{R}_{2}^{3}$ where $H_{1}^{2}$ are the vectors $x$ with $x \cdot x=-1$ in $\mathbb{R}_{2}^{3}$ and $N$ is the oriented unit normal field to $f$.

Proof. Assume first that $N$ exists. Since $d f$ is pointwise injective and

$$
d f(U)=(* d f)\left(J^{\prime} U\right)=(N d f)\left(J^{\prime} U\right)=N N d f(U),
$$

we have $N^{2}=1$. We see that $N$ cannot be \pm 1 , so that $N$ is purely imaginary and a map into $H_{1}^{2}$. Next we would like to see that $N$ is normal to the surface. Note that $-\bar{N}=N$, so that $\overline{d f\left(J^{\prime} U\right)}=\overline{d f(U)} \bar{N}$ gives $-d f\left(J^{\prime} U\right)=d f(U) N$ so that $-N d f(U)=d f(U) N$ and

$$
N d f(U)+d f(U) N=0=-2 N \cdot d f(U),
$$

so that $N$ is a normal field.

Conversely, suppose $f: M_{1}^{2} \rightarrow \mathbb{R}_{2}^{3}$ is a conformal immersion of a Lorentzian surface and assume that $N: M_{1}^{2} \rightarrow H_{1}^{2}$ is the timelike unit normal field to $f$. We need to show that

$$
d f\left(J^{\prime} U\right)=N d f(U)=-N \times d f(U) .
$$

Equivalently we must show that for the null coordinates $\{x, y\}$,

$$
d f(-\partial x)=-N \times f_{x}, \quad d f(\partial y)=-N \times f_{y} .
$$

This is so because $\operatorname{Det}\left[f_{s} / \mu, f_{t} / \mu, N\right]=1$, where $\mu^{2}=f_{s} \cdot f_{s}=-f_{t} \cdot f_{t}=f_{x} \cdot f_{y}$ and $f_{s} \cdot f_{t}=0$.

Lemma 2.3 (Fundamental Lemma). Let $U$ be a Lorentzian subspace in $\mathbb{H}^{\prime}$.

1. There are $L, R \in \mathbb{H}^{\prime}$ such that

$$
L^{2}=R^{2}=1, \quad L U=U=U R, \quad U=\left\{x \in \mathbb{H}^{\prime} \mid L x R=-x\right\} .
$$

$L, R$ are unique up to sign.

2. If $L, U, R$ are as above, and $U \subset \operatorname{Im} \mathbb{H}^{\prime}$, then $L=R$ is the normal vector in $\mathbb{R}_{2}^{3}$.

3. If $L, R \in \mathbb{H}^{\prime}$ with $L^{2}=1=R^{2}$ then $U=\left\{x \in \mathbb{H}^{\prime} \mid L x R=-x\right\}$ and $U^{\perp}=\left\{x \in \mathbb{H}^{\prime} \mid L x R=x\right\}$ are orthogonal real two-dimensional subspaces.

Proof. 1. If $1 \in U$, let $a \in U \subset \operatorname{Im} \mathbb{H}^{\prime}$, so that $a \cdot a=-1$ and $a \cdot 1=0$, keeping in mind that $U=\operatorname{span}\{1, a\}$ is Lorentzian. We can see that one possible choice is $L=a$ and $R=-a$. Indeed, $L^{2}=R^{2}=$ $a^{2}=-a \cdot a=1 ; L U=\{a(v+u a)=v a+u \mid u, v \in \mathbb{R}\}=U$, and 
$U R=\{(u a+v)(-a)=-u-v a\} \in U$, for $u, v \in \mathbb{R}$. Finally we see that

$$
a(u a+v)(-a)=(u+v a)(-a)=-u a-v,
$$

so that $U \subset\left\{x \in \mathbb{H}^{\prime} \mid L x R=-x\right\}$. To see the other containment, take $b, c \in \mathbb{R}_{2}^{3}$ so that $1, a, b, c$ is an orthonormal basis with $b \cdot b=1=-c \cdot c$. Then $a(u+v a+w b+x c)(-a)=-u-a v+w b+x c$, assuming that $a \times b=c$.

To see uniqueness, we see that $L 1=L \in U$, so that $L=u+v a$. Calculating $L^{2}=(u+v a)^{2}=1$ gives $u^{2}+v^{2}+2 u v a=1$, yielding $L=1$ or $L=a$, so $L=a$.

If $U$ is arbitrary, write $U=\operatorname{span}\left\{x, x^{\perp}\right\}$ with $x \cdot x=1, x^{\perp} \cdot x^{\perp}=-1$ and $x \cdot x^{\perp}=0$. If we let, as [1] does, $\tilde{U}=x^{-1} U$ then $a=x^{-1} x^{\perp}$ and $L=x \tilde{L} x^{-1}=x^{\perp} x^{-1}, R=\tilde{R}=-x^{-1} x^{\perp}$.

2. Now let $U=\operatorname{span}\left\{x, x^{\perp}\right\}$ be a Lorentzian subspace of $\operatorname{Im} \mathbb{H}^{\prime}$. Then

$$
L=x^{\perp} x^{-1}=-x^{\perp} \times x^{-1}=x^{-1} \times x^{\perp}=R:=-n
$$

where $n$ is the oriented timelike unit normal vector to the plane in $\operatorname{Im} \mathbb{H}^{\prime}$.

3 . We know that $x^{\perp} x^{-1} x^{\perp} x^{-1}=1=x^{-1} x^{\perp} x^{-1} x^{\perp}$, so that

$$
L\left(u x+b x^{\perp}\right) R=x^{\perp} x^{-1}\left(u x+b x^{\perp}\right)\left(-x^{-1} x^{\perp}\right)=-u x-b x^{\perp} .
$$

Now, $\sigma(x)=L x R$ is an orthogonal transformation, in fact, $L x R \cdot L x R=$ $\bar{R} \bar{x} \bar{L} L x R=x \cdot x$. If $L x R=-x$ and $L y R=y$ then $x \cdot y=-L x R \cdot L y R=-x$. $y=0$. Conversely, if $L x R=-x$ and $x \cdot y=0$, then $L x R \cdot L y R=0=x \cdot L y R$, so that $L y R$ is in the orthogonal complement of the -1 eigenspace. Since $\sigma^{2}=\mathrm{Id}$ is one-to-one, the +1 eigenspace is the orthogonal complement.

Take an immersed surface $f: M_{1}^{2} \rightarrow \mathbb{H}^{\prime}$ with conformal coordinates $\{s, t\}$, so that $f_{s} \cdot f_{s}>0, f_{t} \cdot f_{t}+f_{s} \cdot f_{s}=0$ and $f_{s} \cdot f_{t}=0$. Let $U=$ $\operatorname{span}\left\{f_{s} / \mu, f_{t} / \mu\right\}$. Then $L=f_{t} f_{s}^{-1}, R=-f_{s}^{-1} f_{t}$. Any normal vector $N$ to the surface must satisfy $L N R=N$ or

$$
-f_{s}^{-1} N f_{s}^{-1}=f_{t}^{-1} N f_{t}^{-1} \text {. }
$$

We will derive an equivalent condition for $f_{s} \cdot f_{t}=0$. Note that

$$
\begin{array}{rll}
f_{s} \cdot f_{t}=0 \quad \text { iff } & \bar{f}_{t} f_{s}+\bar{f}_{s} f_{t}=0 \\
& \text { iff } \quad\left(f_{t} \cdot f_{t}\right) f_{t}^{-1} f_{s}+\left(f_{s} \cdot f_{s}\right) f_{s}^{-1} f_{t}=0 \\
& \text { iff } \quad\left(f_{t} \cdot f_{t}\right) f_{t}^{-1} f_{s}=-\left(f_{s} \cdot f_{s}\right) f_{s}^{-1} f_{t} .
\end{array}
$$

We know that in this case $f_{s} \cdot f_{s}, f_{t} \cdot f_{t} \neq 0$, so we can take the inverse of both sides of the last equation and obtain

$$
\frac{f_{s}^{-1} f_{t}}{f_{t} \cdot f_{t}}+\frac{f_{t}^{-1} f_{s}}{f_{s} \cdot f_{s}}=0
$$

If $f_{s} \cdot f_{s}+f_{t} \cdot f_{t}=0$, then

$$
f_{s}^{-1} f_{t}=f_{t}^{-1} f_{s} \quad \text { or } \quad f_{t} f_{s}^{-1}=f_{s} f_{t}^{-1} .
$$


Conversely, if $f_{t} f_{s}^{-1}=f_{s} f_{t}^{-1}$, then

$$
\frac{f_{t} \bar{f}_{s}}{f_{s} \cdot f_{s}}-\frac{f_{s} \bar{f}_{t}}{f_{t} \cdot f_{t}}=0
$$

Since $f_{t} \bar{f}_{s}$ is not real, this implies that $f_{s} \cdot f_{t}=0$ and $f_{s} \cdot f_{s}+f_{t} \cdot f_{t}=0$.

DEFINITION 2.1. If $\{s, t\}$ are conformal coordinates on the immersion $f: M_{1}^{2} \rightarrow \mathbb{R}_{2}^{4} \cong \mathbb{H}^{\prime}$, we say the immersion is real isothermic if $f_{s t}^{\perp}=0$ and complex isothermic if $\left(f_{s s}+f_{t t}\right)^{\perp}=0$. This corresponds to having all the shape operators diagonalizable either over $\mathbb{R}$ or over $\mathbb{C}$.

TheOREM 2.1. Let $f: M_{1}^{2} \rightarrow \mathbb{R}_{2}^{4} \cong \mathbb{H}^{\prime}$ with conformal coordinates $\{s, t\}$. If $f$ is real isothermic, then $\psi^{*}:=f_{s}^{-1} d s+f_{t}^{-1} d t$ is a closed form, and if $\psi^{*}=d f^{*}$ locally, then $f^{*}$ is also real isothermic.

If $f$ is complex isothermic, then $\phi^{*}:=f_{s}^{-1} d t+f_{t}^{-1} d s$ is a closed form, and if $\phi^{*}=d f^{*}$ locally, then $f^{*}$ is also complex isothermic.

Proof. First we check that the forms are closed. In the real case we only need to use (2.3):

$$
\begin{aligned}
d \psi^{*} & =-f_{s}^{-1} d f_{s} f_{s}^{-1} \wedge d s-f_{t}^{-1} d f_{t} f_{t}^{-1} \wedge d t \\
& =-f_{t}^{-1} f_{t s} f_{t}^{-1} d s \wedge d t+f_{s}^{-1} f_{s t} f_{s}^{-1} d s \wedge d t \\
& =\left(-f_{t}^{-1}\left(a f_{s}+b f_{t}\right) f_{t}^{-1} d s \wedge d t+f_{s}^{-1}\left(a f_{s}+b f_{t}\right) f_{s}^{-1}\right) d s \wedge d t \\
& =\left(-a f_{s}^{-1}-b f_{t}^{-1}+a f_{s}^{-1}+b f_{t}^{-1}\right) d s \wedge d t=0 .
\end{aligned}
$$

For the complex isothermic case we need equation $(2.2)$ as well:

$$
\begin{aligned}
d \phi^{*}= & -f_{s}^{-1} f_{s s} f_{s}^{-1} d s \wedge d t+f_{t}^{-1} f_{t t} f_{t}^{-1} d s \wedge d t \\
= & -f_{s}^{-1}\left(b f_{s}+a f_{t}+e_{1} N_{1}+e_{2} N_{2}\right) f_{s}^{-1} d s \wedge d t \\
& +f_{t}^{-1}\left(b f_{s}+a f_{t}-e_{1} N_{1}-e_{2} N_{2}\right) f_{t}^{-1} d s \wedge d t \\
= & \left(-b f_{s}^{-1}-a f_{t}^{-1}-e_{1} f_{s}^{-1} N_{1} f_{s}^{-1}-e_{2} f_{s}^{-1} N_{2} f_{s}^{-1}\right. \\
& \left.+b f_{s}^{-1}+a f_{t}^{-1}-e_{1} f_{t}^{-1} N_{1} f_{t}^{-1}-e_{2} f_{t}^{-1} N_{2} f_{t}^{-1}\right) d s \wedge d t=0 .
\end{aligned}
$$

Next we show that $f^{*}$ is isothermic. In the real isothermic case we have

$$
d f^{*}=f_{s}^{-1} d s+f_{t}^{-1} d t
$$

so that

$$
f_{s}^{*}=f_{s}^{-1} \quad \text { and } \quad f_{t}^{*}=f_{t}^{-1} .
$$

We must show that

$$
\begin{aligned}
f_{t}^{*} f_{s}^{*-1} & =f_{s}^{*} f_{t}^{*-1}, \\
f_{s t}^{*} & =\alpha f_{s}^{*}+\beta f_{t}^{*} .
\end{aligned}
$$

Clearly (2.6) holds by (2.3). For (2.7),

$$
\begin{aligned}
\left(f_{t}^{*}\right)_{s} & =\left(f_{t}^{-1}\right)_{s}=-f_{t}^{-1} f_{t s} f_{t}^{-1}=-f_{t}^{-1}\left(a f_{s}+b f_{t}\right) f_{t}^{-1} \\
& =-a f_{s}^{-1}-b f_{t}^{-1}=-a f_{s}^{*}-b f_{t}^{*}
\end{aligned}
$$


In the complex isothermic case,

$$
f_{s}^{*}=f_{t}^{-1} \quad \text { and } \quad f_{t}^{*}=f_{s}^{-1} .
$$

We can easily see that

$$
f_{t}^{*} f_{s}^{*-1}=f_{s}^{*} f_{t}^{*-1} .
$$

Finally, we must see that $f_{s s}^{*}+f_{t t}^{*}$ is purely tangential:

$$
\begin{aligned}
& f_{s s}^{*}+f_{t t}^{*}=\left(f_{t}^{-1}\right)_{s}+\left(f_{s}^{-1}\right)_{t}=-f_{t}^{-1} f_{s t} f_{t}^{-1}-f_{s}^{-1} f_{t s} f_{s}^{-1} \\
& \begin{aligned}
=-f_{s}^{-1}\left(a f_{s}+b f_{t}+c_{1} N_{1}+c_{2} N_{2}\right) f_{s}^{-1}-f_{t}^{-1}\left(a f_{s}+b f_{t}\right. & \left.+c_{1} N_{1}+c_{2} N_{2}\right) f_{t}^{-1} \\
& =-2 a f_{s}^{-1}-2 b f_{t}^{-1} .
\end{aligned}
\end{aligned}
$$

Note that $\phi^{* *}=f_{t}^{*-1} d s+f_{s}^{*-1} d t=f_{s} d s+f_{t} d t=d f$, so that $f^{* *}=f$ and the same is true for $f$ real isothermic.

We can also see that, in both cases, $d f \wedge d f^{*}=0$. Indeed, in the real isothermic case,

$d f \wedge d f^{*}=\left(f_{s} d s+f_{t} d t\right) \wedge\left(f_{s}^{-1} d s+f_{t}^{-1} d t\right)=\left(f_{s} f_{t}^{-1}-f_{t} f_{s}^{-1}\right)(d s \wedge d t)=0$, while in the complex isothermic case, $d f \wedge d f^{*}=\left(f_{s} d s+f_{t} d t\right) \wedge\left(f_{t}^{-1} d s+f_{s}^{-1} d t\right)=\left(f_{s} f_{s}^{-1}-f_{t} f_{t}^{-1}\right)(d s \wedge d t)=0$.

Proposition 2.1. If $g: M_{1}^{2} \rightarrow \mathbb{H}^{\prime}$ is isothermic, then $\bar{g}$ is isothermic. Furthermore, $f$ and $f^{c}=\bar{f}^{*}$, as given in Theorem 2.1, share the same tangent planes.

Proof. We note that $x \cdot y=\bar{x} \cdot \bar{y}$, so that the normal space to the image of $\bar{g}$ is the conjugate of the normal space to $g$. Thus, for example, if $g$ is real isothermic, so that $\left[g_{s t}\right]^{\perp}=0$, then $\left[\bar{g}_{s t}\right]^{\perp}=0$ and $\bar{g}$ is real isothermic.

The claim about the tangent planes depends on the formula for the inverse in $\mathbb{H}^{\prime}$. We note that, by 2.5 or $(2.8)$ we have $f_{t}^{c}=\left(\bar{f}^{*}\right)_{t}=\left(\bar{f}_{t}\right)^{-1}$ or $\left(\bar{f}_{s}\right)^{-1}$. This is either

$$
\frac{f_{t}}{f_{t} \cdot f_{t}} \text { or } \frac{f_{s}}{f_{s} \cdot f_{s}} \text {. }
$$

In either case, $f^{c}$ and $f$ have the same tangent spaces.

Hence we can now set the following definition.

Definition 2.2. Using the definition of $f^{*}$ in Theorem 2.1. $f^{c}=\bar{f}^{*}$ is the Christoffel transform of $f$ and the two surfaces $\left\{f, f^{c}\right\}$ are called a Christoffel pair.

TheOREM 2.2. If $f, g: M_{1}^{2} \rightarrow \mathbb{H}^{\prime}$ are timelike surfaces satisfying $d f \wedge$ $d g=0=d g \wedge d f$, then both are isothermic and $g= \pm f^{*}$. Moreover, $f, \bar{g}$ constitute a Christoffel pair. 
Proof. Choose conformal parameters $\{s, t\}$ for $f$, so that

$$
f_{t} \bar{f}_{s} \in \operatorname{Im} \mathbb{H}^{\prime}, \quad f_{s} \cdot f_{s}+f_{t} \cdot f_{t}=0 .
$$

Set $f_{s} \cdot f_{s}=E$. Examining the two equations in the hypothesis, we find that

$$
f_{t} g_{s}=f_{s} g_{t} \text { and } g_{t} f_{s}=g_{s} f_{t} .
$$

Next we see that $f_{t} g_{t}$ commutes with $f_{s} \bar{f}_{t}$ :

$$
f_{t}\left(g_{t} f_{s}\right) \bar{f}_{t}=f_{t} g_{s} f_{t} \bar{f}_{t}=-E f_{s} g_{t}=f_{s}\left(\bar{f}_{t} f_{t}\right) g_{t} .
$$

If a (split-)quaternion $v$ commutes with an imaginary quaternion $u$, then $v \in \operatorname{span}\{1, u\}$. Here, then, $f_{t} g_{t} \in \operatorname{span}\left\{1, f_{s} \bar{f}_{t}\right\}$. Set $f_{t} g_{t}=a+b f_{s} \bar{f}_{t}$, so that

$$
g_{t}=a f_{t}^{-1}-b E f_{s}^{-1} \text {. }
$$

In the same way we can see that $f_{s} g_{s}$ commutes with $f_{t} \bar{f}_{s}$, so that

$$
g_{s}=c f_{s}^{-1}+d E f_{t}^{-1} \text {. }
$$

Using (2.10), 2.11) and (2.12), we see that, in fact,

$$
\begin{aligned}
& g_{t}=\alpha f_{t}^{-1}+\beta f_{s}^{-1}, \\
& g_{s}=\beta f_{t}^{-1}+\alpha f_{s}^{-1} .
\end{aligned}
$$

Next we look at the conditions for the integrability of $g$. Using 2.2 and (2.3), $g_{t s}=g_{s t}$ yields

$$
\begin{aligned}
\alpha_{s} & =\beta_{t}, \\
\beta_{s} & =\alpha_{t}, \\
2 \alpha f_{s t}^{\perp}-\beta\left(f_{s s}+f_{t t}\right)^{\perp} & =0 .
\end{aligned}
$$

We will now define a conformal change of coordinates, depending on the values $\alpha$ and $\beta$. For any functions $a, b$ with $a^{2} \neq b^{2}$ set

$$
\begin{aligned}
d u & =a d t+b d s, & \frac{\partial}{\partial u} & =\frac{a}{a^{2}-b^{2}} \frac{\partial}{\partial t}-\frac{b}{a^{2}-b^{2}} \frac{\partial}{\partial s}, \\
d v & =b d t+a d s, & \frac{\partial}{\partial v} & =-\frac{b}{a^{2}-b^{2}} \frac{\partial}{\partial t}+\frac{a}{a^{2}-b^{2}} \frac{\partial}{\partial s} .
\end{aligned}
$$

Given $\{u, v\}$ we see that

$$
\begin{aligned}
\left(f_{u u}+f_{v v}\right)^{\perp} & =-\frac{4 a b}{\left(a^{2}-b^{2}\right)^{2}} f_{t s}^{\perp}+\frac{a^{2}+b^{2}}{\left(a^{2}-b^{2}\right)^{2}}\left(f_{t t}+f_{s s}\right)^{\perp}, \\
f_{u v}^{\perp} & =\frac{a^{2}+b^{2}}{\left(a^{2}-b^{2}\right)^{2}}\left(f_{t s}\right)^{\perp}-\frac{a b}{\left(a^{2}-b^{2}\right)^{2}}\left(f_{t t}+f_{s s}\right)^{\perp} .
\end{aligned}
$$

There are two cases to consider.

1. $|\alpha|>|\beta|$, i.e., $|\alpha|+\beta k^{\prime}$ is in quadrant I of $\mathbb{C}^{\prime}$ (see the appendix.) If $\alpha>0$ we solve $\left(a+b k^{\prime}\right)^{2}=\alpha+\beta k^{\prime}$. Explicitly, we have $a=\sqrt{\alpha} \cos (\rho), b=$ 
$\sqrt{\alpha} \sin (\rho)$, where $\sin (2 \rho)=\beta / \alpha$. This gives $\alpha^{2}-\beta^{2}=\left(a^{2}+b^{2}\right)^{2}-4 a^{2} b^{2}=$ $\left(a^{2}-b^{2}\right)^{2}$. In this case

$$
2 f_{u v}^{\perp}=\frac{2 \alpha}{\alpha^{2}-\beta^{2}} f_{t s}^{\perp}-\frac{\beta}{\alpha^{2}-\beta^{2}}\left(f_{t t}+f_{s s}\right)^{\perp}=0,
$$

and $f$ is real isothermic.

If $\alpha<0$, solve $\left(a+b k^{\prime}\right)^{2}=-\alpha-\beta k^{\prime}$, which lies in quadrant I and again we see that $f$ is real isothermic.

2. $|\beta|>|\alpha|$. In this case, if $\beta>0$, solve $\left(a+b k^{\prime}\right)^{2}=\beta+\alpha k^{\prime}$. Here we have $a^{2}+b^{2}=\beta$ and $2 a b=\alpha$, and find

$$
\left(f_{u u}+f_{v v}\right)^{\perp}=-\frac{2 \alpha}{\alpha^{2}-\beta^{2}} f_{t s}^{\perp}+\frac{\beta}{\alpha^{2}-\beta^{2}}\left(f_{t t}+f_{s s}\right)^{\perp}=0,
$$

so that $f$ is complex isothermic. If $\beta<0$ we solve $\left(a+b k^{\prime}\right)^{2}=-\beta-\alpha k^{\prime}$, with the same result.

We next see that $g$ is $\pm f^{*}$.

In the real case we show that

$$
d g=g_{u} d u+g_{v} d v=f_{u}^{-1} d u+f_{v}^{-1} d v,
$$

i.e., $g_{u} f_{u}=1=g_{v} f_{v}$. We know that

$$
g_{u}=\frac{a g_{t}-b g_{s}}{a^{2}-b^{2}} \text { and } g_{v}=\frac{-b g_{t}+a g_{s}}{a^{2}-b^{2}} .
$$

As above, letting $\alpha=\tau\left(a^{2}+b^{2}\right)$ and $\beta=2 \tau a b$ with $\tau= \pm 1$ we get

$$
f_{u} g_{u}=\left(\frac{a f_{t}-b f_{s}}{a^{2}-b^{2}}\right)\left(\frac{a\left(\alpha f_{t}^{-1}+\beta f_{s}^{-1}\right)-b\left(\beta f_{t}^{-1}+\alpha f_{s}^{-1}\right)}{a^{2}-b^{2}}\right)=\tau .
$$

We see that $f_{v} g_{v}=\tau$ in the same way.

For the complex case we want to see that $g_{u} f_{v}=\tau=g_{v} f_{u}$, using $\alpha=$ $2 \tau a b$ and $\beta=\tau\left(a^{2}+b^{2}\right)$. We have

$$
f_{u} g_{v}=\left(\frac{a f_{t}-b f_{s}}{a^{2}-b^{2}}\right)\left(\frac{-b\left(\alpha f_{t}^{-1}+\beta f_{s}^{-1}\right)+a\left(\beta f_{t}^{-1}+\alpha f_{s}^{-1}\right.}{a^{2}-b^{2}}\right)=\tau
$$

and can also see easily that $g_{v} f_{u}=\tau$.

Next we give two examples which are codimension two and which do not have positive definite analogs.

ExAmple 2.1. The unit complex circle

$$
f(s, t)=(\cos (s) \cosh (t), \sin (s) \cosh (t),-\sin (s) \sinh (t), \cos (s) \sinh (t))
$$

is complex isothermic. We have

$$
\begin{aligned}
& f_{t}(s, t)=(\cos (s) \sinh (t), \sin (s) \sinh (t),-\sin (s) \cosh (t), \cos (s) \cosh (t)), \\
& f_{s}(s, t)=(-\sin (s) \cosh (t), \cos (s) \cosh (t),-\cos (s) \sinh (t),-\sin (s) \sinh (t)) .
\end{aligned}
$$

Thus we see that $f_{s} \cdot f_{s}=1, f_{t} \cdot f_{t}=-1$, and $f_{s} \cdot f_{t}=0$. 
$N_{1}=(\cos (s) \cosh (t), \sin (s) \cosh (t),-\sin (s) \sinh (t), \cos (s) \sinh (t))$,

$N_{2}=(\sin (s) \sinh (t),-\cos (s) \sinh (t), \cos (s) \cosh (t), \sin (s) \cosh (t))$,

$f_{t}^{-1}=f_{s}^{*}=(-\cos (s) \sinh (t), \sin (s) \sinh (t),-\sin (s) \cosh (t), \cos (s) \cosh (t))$,

$f_{s}^{-1}=f_{t}^{*}=(-\sin (s) \cosh (t),-\cos (s) \cosh (t), \cos (s) \sinh (t), \sin (s) \sinh (t))$,

$f^{*}(s, t)=(-\sin (s) \sinh (t),-\cos (s) \sinh (t), \cos (s) \cosh (t), \sin (s) \cosh (t))$,

Hence the Christoffel transform $f^{c}$ is given by

$f^{c}(s, t)=(-\sin (s) \sinh (t), \cos (s) \sinh (t),-\cos (s) \cosh (t),-\sin (s) \cosh (\mathrm{t}))$.

EXAMPLE 2.2. The immersion

$$
f(s, t)=\left(s^{2}+t^{2}, s, t, s^{2}+t^{2}\right)
$$

is real isothermic. We have

$$
\begin{aligned}
f_{s}(s, t) & =(2 s, 1,0,2 s), \\
f_{t}(s, t) & =(2 t, 0,1,2 t), \\
f_{t}^{-1} & =f_{t}^{*}=(-2 t, 0,1,2 t), \\
f_{s}^{-1} & =f_{s}^{*}=(2 s,-1,0,-2 s), \\
N_{1} & =\left(\frac{2 s^{2}-2 t^{2}+1 / 2}{\sqrt{2}}, \sqrt{2} s,-\sqrt{2} t, \frac{2 s^{2}-2 t^{2}+3 / 2}{\sqrt{2}}\right), \\
N_{2} & =\left(\frac{-2 s^{2}+2 t^{2}+3 / 2}{\sqrt{2}},-\sqrt{2} s, \sqrt{2} t, \frac{-2 s^{2}+2 t^{2}+1 / 2}{\sqrt{2}}\right), \\
f^{*}(s, t) & =\left(s^{2}-t^{2},-s, t,-s^{2}+t^{2}\right) .
\end{aligned}
$$

The Christoffel transform $f^{c}$ is given by

$$
f^{c}(s, t)=\left(s^{2}-t^{2}, s,-t, s^{2}-t^{2}\right) .
$$

3. Timelike surfaces in $\mathbb{R}_{2}^{3}$. We want to look at the special case when our surface is contained in the imaginary quaternions. Let $\{s, t\}$ be isothermal coordinates in $\operatorname{Im} \mathbb{H}^{\prime}$, with $f_{s} \cdot f_{s}=\mu^{2}$ and $N \cdot N=-1$. The fundamental equations of the immersion $f$ are given by

$$
\begin{aligned}
f_{t t} & =\frac{\mu_{t}}{\mu} f_{t}+\frac{\mu_{s}}{\mu} f_{s}+a N, \\
f_{t s} & =\frac{\mu_{s}}{\mu} f_{t}+\frac{\mu_{t}}{\mu} f_{s}+b N, \\
f_{s s} & =\frac{\mu_{t}}{\mu} f_{t}+\frac{\mu_{s}}{\mu} f_{s}+c N, \\
N_{t} & =-\frac{a}{\mu^{2}} f_{t}+\frac{b}{\mu^{2}} f_{s}, \\
N_{s} & =-\frac{b}{\mu^{2}} f_{t}+\frac{c}{\mu^{2}} f_{s} .
\end{aligned}
$$


Thus, the shape operator corresponding to $N$ is

$$
A_{N}=\left(\begin{array}{cc}
a / \mu^{2} & +b / \mu^{2} \\
-b / \mu^{2} & -c / \mu^{2}
\end{array}\right) .
$$

In [7] Inoguchi and Toda give a definition of the Hopf differentials using null coordinates. We give a similar definition using the associated isothermal coordinates via a split-complex variable. Let $z^{\prime}=s+k^{\prime} t: M_{1}^{2} \rightarrow \mathbb{C}^{\prime}$ for isothermal coordinates $\{s, t\}$ on $M_{1}^{2}$. On $T^{\mathbb{C}^{\prime}} M$ we define

$$
\frac{\partial}{\partial z^{\prime}}=\frac{1}{2}\left(\frac{\partial}{\partial s}+k^{\prime} \frac{\partial}{\partial t}\right) \text {. }
$$

We need the + sign to be consistent with $d z^{\prime}\left(\partial / \partial z^{\prime}\right)=\left(d s+k^{\prime} d t\right)\left(\partial / \partial z^{\prime}\right)=1$. We extend the metric on $\mathbb{R}_{2}^{4}$ to $\mathbb{C}^{\prime 2}$ bilinearly.

Lemma 3.1. The coordinates $\{s, t\}$ are conformal coordinates for an immersion $f: M_{1}^{2} \rightarrow \mathbb{H}^{\prime}$ iff $z^{\prime}=s+k^{\prime} t$ are isotropic coordinates for $f$.

Proof. We calculate

$$
f_{z^{\prime}} \cdot f_{z^{\prime}}=\frac{1}{4}\left(f_{s} \cdot f_{s}+f_{t} \cdot f_{t}+2 k^{\prime} f_{t} \cdot f_{s}\right) .
$$

The isotropy condition does not determine the sign of $f_{s} \cdot f_{s}$.

Continuing, we see that

$$
f_{z^{\prime} z^{\prime}}=\frac{1}{4}\left(f_{s s}+f_{t t}\right)+\frac{1}{2} k^{\prime} f_{t s} .
$$

Thus $\{s, t\}$ are real conjugate parameters iff the imaginary part of $f_{z^{\prime} z^{\prime}}$ is tangential, while they are $i$-conjugate parameters iff the real part of $f_{z^{\prime} z^{\prime}}$ is tangential. Here $i$-conjugate means that $A_{N}(\partial s+i \partial t) \cdot \partial s-i \partial t=0$, where $N$ is a unit normal vector field.

Definition 3.1. For a normal vector field $N: M_{1}^{2} \rightarrow H_{1}^{2}$ the $H o p f$ differential $Q_{N}$ is $\left(f_{z^{\prime} z^{\prime}} \cdot N\right) d z^{\prime 2}$, a quadratic differential ([4]).

Thus if we have real conjugate parameters then $Q_{N}$ is a real multiple of $d z^{\prime 2}$, while for $i$-conjugate parameters, $Q_{N}$ is a real multiple of $k^{\prime} d z^{\prime 2}$, i.e., a pure split-imaginary multiple of $d z^{\prime 2}$.

We begin with the timelike version of Lemma 3.3.27 in [6], for which $H$ and $N$ denote the mean curvature and the Gauss map corresponding to $f$.

Lemma 3.2. If $f, f^{c}: M_{1}^{2} \rightarrow \mathbb{R}_{2}^{3}$ form a Christoffel pair, then, with $N^{c}=-N$,

$$
d N+H d f=H^{c} d f^{c} .
$$

Proof. Using (3.4), 3.5) we see that

$$
d N+H d f=\left(\frac{-a-c}{2 \mu^{2}} f_{t}+\frac{b}{\mu^{2}} f_{s}\right) d t+\left(\frac{a+c}{2 \mu^{2}} f_{s}-\frac{b}{\mu^{2}} f_{t}\right) d s .
$$


We will only consider the case where the immersions are complex isothermic, so that $a+c=0$. The equation (3.6) reduces to

$$
d N+H d f=\frac{b}{\mu^{2}} f_{s} d t-\frac{b}{\mu^{2}} f_{t} d s=\lambda^{c} d f^{c}
$$

for some $\lambda^{c}$, since in this case,

$$
d f^{c}=\frac{f_{s}}{\mu^{2}} d t-\frac{f_{t}}{\mu^{2}} d s .
$$

Following Hertrich-Jeromin, we see that $-d N+H^{c} d f^{c}=\lambda d f$, and the result follows.

Thus, in either case we have $d f^{c}=\rho(d N+H d f)$.

LEMMA 3.3. Every immersion of a timelike surface $M_{1}^{2}$ in $\mathbb{R}_{2}^{3}$ with normal field $N$ and mean curvature $H$ satisfies

$$
d f \wedge(d N+H d f)=0 .
$$

Proof. For an arbitrary conformal coordinate system $\{s, t\}$,

$$
\begin{aligned}
d f \wedge(d N+H d f)= & \frac{1}{\mu^{2}}\left(-b f_{t} f_{t}+c f_{t} f_{s}+a f_{s} f_{t}-b f_{s} f_{s}\right) d t \wedge d s \\
& +\frac{a-c}{2 \mu^{2}}\left(f_{t} f_{s}-f_{s} f_{t}\right)(d t \wedge d s) .
\end{aligned}
$$

We can let $f_{t} \times f_{s}=\mu^{2} N$, so that, using 2.1), our equation is

$$
-b-c N+a N+b+\frac{a-c}{2}(-2 N)=0 \text {. }
$$

Just as in [6] we have:

Corollary 3.1. A surface $f: M_{1}^{2} \rightarrow \mathbb{R}_{2}^{3}$ is isothermic iff there is locally an integrating factor $\rho: M_{1}^{2} \rightarrow \mathbb{R}$, so that $d[\rho(d N+H d f)]=0$.

Using Lemma 3.2, 3.3 and Corollary 3.1 we see that whenever one of the timelike surfaces in $\mathbb{R}_{2}^{3}$ of a Christoffel pair is a minimal surface the other is totally umbilic, namely a scaling of its Gauss map, and vice versa. Moreover, the Christoffel transform of a timelike surface of constant mean curvature $H \neq 0$ is its parallel surface $f+(1 / H) N$ of constant mean curvature $H^{c}=H$. In particular the integrating factor can be chosen to be constant in both cases.

The next proposition shows how the split-complex numbers arise naturally when looking at timelike surfaces in $\mathbb{R}_{2}^{4}$.

Proposition 3.1. Let $f, f^{c}: M_{1}^{2} \rightarrow \mathbb{R}_{2}^{3}$ be a Christoffel pair of isothermic surfaces and $N: M_{1}^{2} \rightarrow H_{1}^{2}=\{x \mid x \cdot x=-1\}$. Identify the trivial normal bundle $N M$ of $f: M_{1}^{2} \rightarrow \mathbb{H}^{\prime}$ with the trivial $\mathbb{C}^{\prime}$ bundle $M \times \mathbb{C}^{\prime}$, so that $N_{p} M=\operatorname{span}\{1, N(p)\} \cong \mathbb{C}^{\prime}=\operatorname{span}\left\{1, k^{\prime}\right\}$. The map

$$
q=d f \cdot d f^{c}: T M \rightarrow N M
$$


is a split-holomorphic quadratic differential. The Hopf differential is a real multiple of $q$ if the immersions are real isothermic, and a pure imaginary multiple if the immersions are complex isothermic.

Proof. Set $N=\frac{f_{t}}{\mu} \times \frac{f_{s}}{\mu}$. Then $f_{t} f_{s}^{-1}=N=f_{s} f_{t}^{-1}$. In the real isothermic case

$$
d f \cdot d f^{c}=-\left[d t^{2}+d s^{2}+2 N d t d s\right]=-\left(d s+k^{\prime} d t\right)^{2}=-d z^{\prime 2} .
$$

In the complex isothermic case

$$
d f \cdot d f^{c}=-N\left(d t^{2}+d s^{2}\right)-2 d t d s=-k^{\prime} d z^{\prime 2} .
$$

4. Appendix: Polar coordinates for $\mathbb{C}^{\prime}$. The split-complex numbers are $\mathbb{C}^{\prime}=\left\{w=a+b k^{\prime} \mid a, b \in \mathbb{R}, k^{\prime 2}=1\right\}$. We are interested in square roots of split-complex numbers. For convenience, we break the split-complex plane into the quadrants determined by the null lines $\operatorname{Re}(w)= \pm \operatorname{Im}(w)$. Let quadrant I be defined by $\left\{a+b k^{\prime} \in \mathbb{C}^{\prime}|| a|>| b \mid, a>0\right\}$, with quadrants II, III and IV numbered counter-clockwise from there. Numbers in quadrant I can be written, with $r>0$, as

$$
w=r e^{\theta k^{\prime}}=r \cosh (\theta)+r \sinh (\theta) k^{\prime},
$$

where the second equality follows from the power series expansion of $e^{t}$. Points in quadrant II can be written in the form

$$
r e^{\theta k^{\prime}} k^{\prime}=r \sinh (\theta)+r \cosh (\theta) k^{\prime} .
$$

In the other two quadrants numbers are of the form $-r e^{\theta k^{\prime}}$ and $-r e^{\theta k^{\prime}} k^{\prime}$. Note that to consider all the split-complex numbers we must also look at the null lines of the form $w=a \pm a k^{\prime}$.

Lemma 4.1. A split-complex number has a square root iff it is in quadrant $I$ or is of the form $w=a \pm a k^{\prime}$ with $a \geq 0$.

Proof. Using the polar form, we can see that all squares from the quadrants land in quadrant I, and conversely, every number in quadrant I has a square root. Finally we consider the null lines. If $a>0$, then $\left(-a \pm a k^{\prime}\right)^{2}=$ $2 a^{2} \mp 2 a^{2} k^{\prime}$, so that squares of null elements with negative real part have positive real parts and are null. It is clear that null elements with positive real parts are squares.

Acknowledgments. The second author would like to express his thanks to the Institute of Mathematics and Statistics (IME) at the University of São Paulo S.P. for its hospitality and the State of São Paulo Research Foundation (FAPESP) for financial support during the development of part of this work. 


\section{References}

[1] F. E. Burstall, D. Ferus, K. Leschke, F. Pedit, and U. Pinkall, Conformal Geometry of Surfaces in $S^{4}$ and Quaternions, Lecture Notes in Math. 1772, Springer, 2002.

[2] M. P. Dussan and M. Magid, Timelike isothermic surfaces associated to Grassmannian systems, Documenta Math. 10 (2005), 527-549.

[3] - , - Complex timelike isothermic surfaces and their geometric transformations, Balkan J. Geom. Appl. 11 (2006), 39-53.

[4] S. Erdem, Harmonic maps of Lorentz surfaces, quadratic differentials and paraholomorphicity, Beiträge Algebra Geom. 38 (1997), 19-32.

[5] A. Fujioka and J. Inoguchi, Timelike surfaces with harmonic inverse mean curvature, in: Surveys on Geometry and Integrable Systems, Adv. Stud. Pure Math. 51, Math. Soc. Japan, 2008, 113-141.

[6] U. Hertrich-Jeromin, Introduction to Möbius Differential Geometry, London Math. Soc. Lecture Note Ser. 300, Cambridge Univ. Press, 2003.

[7] J. Inoguchi and M. Toda, Timelike minimal surfaces via loop groups, Acta Appl. Math. 83 (2004), 313-355.

[8] G. Kamberov, F. Pedit and U. Pinkall, Bonnet pairs and isothermic surfaces, Duke Math. J. 92 (1998), 637-644.

[9] P. Libermann, Sur les structures presque quaternioniennes de deuxième espèce, C. R. Acad. Sci. Paris 234 (1952), 1030-1032.

[10] —, Sur les structures presque paracomplexes, ibid. 234 (1952), 2517-2519.

[11] M. Magid, Lorentzian isothermic surfaces, Rocky Mountain J. Math. 35 (2005), 627-640.

[12] - , Lorentzian isothermic surfaces and Bonnet pairs, Ann. Polon. Math. 83 (2004), $129-139$.

[13] T. Weinstein, An Introduction to Lorentz Surfaces, de Gruyter, Berlin, 1996.

M. P. Dussan

Departamento de Matemática - IME

Universidade de São Paulo

CEP: 05508-090

São Paulo S.P., Brazil

E-mail: dussan@ime.usp.br
M. Magid

Department of Mathematics

Wellesley College

Wellesley, MA 02458. U.S.A.

E-mail: mmagid@wellesley.edu

Received 14.12.2009

and in final form 11.6.2010 